\title{
Correction to: Enlightening the black and white: species delimitation and UNITE species hypothesis testing in the Russula albonigra species complex
}

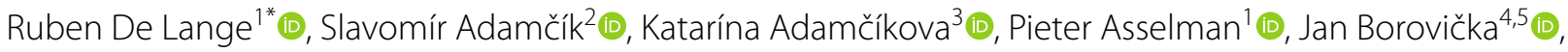 \\ Lynn Delgat ${ }^{1,6}$ (D), Felix Hampe ${ }^{1}$ (i) and Annemieke Verbeken ${ }^{1}$ (1)
}

\section{Correction to: IMA Fungus (2021) 12:20}

https://doi.org/10.1186/s43008-021-00064-0

Following the publication of the original article [1], we were notified of a few inconsistencies between the pdf version and the html version with regards to the Key to the European species of Russula subgen. Compactae (pg. 25).

- In the online version for steps 3(2), 9(8) and 12(10): part of first option was gone and combined with the second option.

- In the online version for step 13(12), both options were present but formed one block of text. "Context" on the second line should be the start of option two.

The original article has now been corrected.

\section{Author details}

${ }^{1}$ Research Group Mycology, Department of Biology, Ghent University, K.L. Ledeganckstraat 35, 9000 Ghent, Belgium. ${ }^{2}$ Institute of Botany, Plant Science and Biodiversity Center, Slovak Academy of Sciences, Dúbravská cesta 9, 845 23 Bratislava, Slovakia. ${ }^{3}$ Institute of Forest Ecology Slovak Academy of Sciences, Akademická 2, 94901 Nitra, Slovakia. ${ }^{4}$ Institute of Geology of the Czech Academy of Sciences, Rozvojová 269, 16500 Prague 6, Czech Republic.

${ }^{5}$ Nuclear Physics Institute of the Czech Academy of Sciences, Hlavní 130, 250

68 Husinec-Řež, Czech Republic. ${ }^{6}$ Meise Botanic Garden, Research Department, Nieuwelaan 38, 1860 Meise, Belgium.

Published online: 04 October 2021

\section{Reference}

1. De Lange et al. Enlightening the black and white: species delimitation and UNITE species hypothesis testing in the Russula albonigra species complex (2021) 12:20. https://doi.org/10.1186/s43008-021-00064-0

\section{Publisher's Note}

Springer Nature remains neutral with regard to jurisdictional claims in published maps and institutional affiliations. 